\title{
Research and Experimental of MRI RF High Power Bridge
}

\author{
Jiaping Zhou ${ }^{1}$ Yingliang $\mathrm{Li}^{2}$ Hua Chen ${ }^{1}$ \\ ${ }^{1}$ Xingyi Normal University for Nationalities, Xingyi, Guizhou,China. \\ ${ }^{2}$ Xingaoyi Medical Equipment Co. Ltd; Ningbo, China.
}

\begin{abstract}
A micro-scale 3dB high power RF (Radio Frequency) balance 4 Ports Bridge of MRI (Magnet Resonator Imaging) is realized with 3D PCB manufacturing and integrated capacity and theoretical calculation in this paper. At first a analysis method is proposed based on transmission line theory of the 1/4-wavelength and the lump and transmission parameters, and the transmisson line length and lump components of capacitors value are gotted for this micro-scale bridge. Then this Micro-Balance Bridge (MBB) manufacturing with FR4 and overall size is $100 \mathrm{~mm} \times 60 \mathrm{~mm} \times 1 \mathrm{~mm}$, and it size reduce $1 / 3$ compared with plane bridge. MBB could be achieved 2 ways amplitude balance is about $\pm 0.1 \mathrm{~dB}$, phase balance is $\pm 1^{\circ}$, insert loss is about $0.3 \mathrm{~dB}$, flatness is not more than $0.01 \mathrm{~dB}$ on the operate frequency of $64 \mathrm{MHZ} \pm 5 \mathrm{MHZ}$. This bridge could be supported input pulse power more than $1000 \mathrm{~W}$ by temperature experiment result. Use MBB integrate into the MRI systems and make imaging experiment and could reduce $20 \%$ imaging time from $1.2 \mathrm{~ms}$ to $900 \mathrm{us}$, and it has good efficiency and homogeneity.
\end{abstract}

\section{Introduction}

The magnetic resonance RF power bridge is splitted one way high power signal to two ways balance signals to polarize the body coil with its phase's difference of 90 degrees. The performance of the device will affect the polarization efficiency of the coil. In order to improve the polarization efficiency of the coil, it is necessary to realize the phase difference of the two ways signal to be accurate to 90 degrees, and the two signals amplitudes are balanced. The isolation port need to ensure that the reflected signal of the coil is completely absorbed, when the condition of load variation, the phase and amplitudes of two ports are kept the balance. Furthermore, the RF coil could achieve the very high transmitter effency in the conditions of VSWR (Voltage Standing Wave Ratio) varation $(1: 1 \sim 1: 5)$. A new 4 ports mixed balance bridge is studied to get the phase and amplitude balance in this paper. In gerneral, banace bridge is a splliter with the isolation ports[1]. For MRI balance bridge, and it has more special requirements:

(1)All components can not contain magnetic materials, and also need meet very high voltage capability.Because the peak power of the RF power amplifier is very large, it can reach the kilowatt level, the bridge need meet the large voltage capability.

(2) General bridges are usually implemented using microwave integrated circuits.But it is in the lower frequency band of MRI operator band, if use this method will lead to large size, high loss, low power bearing capacity etc. If only use the lump parameter components could lead to more parasitic parameters, poor device consistency, and complicated debugging processing.
According to the basic requirements of the magnetic resonance system for balanced bridges, this paper use parts of MMIC to replace the original lumped components of inductors, and also use the capacitors. The bridge has the advantages of simple manufacturing process, wide bandwidth, strong power capacity and small size. After theoretical simulation and experimental research, the device has better performance. Compared with the domestic and international magnetic resonance system transmitting power bridge, the balance bridge has better isolation and balancer. It could be easy integrated with the RF transmitting coil to achieve the entire miniaturized magnetic resonance RF front-end system if further studied. At last,this BB are taken he imaging experiment in MRI system and the image uniformity and polarity are improved compared with the international products of the same type.

\section{Fundamental principles of RF power bridge for MRI experimental verification}

The magnetic resonance RF power bridge is shown in Figure 1. The BB divides RF power amplifier (PA) output signal into two equal amplitudes and their phase difference of 90 . These two signals polarizes the RF coil to achieve uniform RF B1 field. Since the magnetic resonance system has a large load variation which could be varied from a very low load to a full load (VSWR:1:1 1:5), so BB need has very strong isolation more than $20 \mathrm{~dB}$. According to the requirements of the MRI system, P1 is input port, P2 and P3 are output port with the same amplitude andphase difference is 90 degrees, and the $\mathrm{P} 4$ is the isolated. A standard bridge 
consists of 4 transmission lines. The characteristic impedance of $\mathrm{L}_{2}$ and $\mathrm{L}_{1}$ is $Z_{0}, Z_{0} / \sqrt{2}$, and the length is $\lambda / 4$ lines show as fig2. It can be seen that its size is very large when the operating frequency is $64 \mathrm{MHz}$, the length of a single bridge is greater than $1.17 \mathrm{~m}$. It can be seen that the length is very long and then these lengths of microwave lines are realized on a PCB board, considering their power capacity and loss characteristics, if use FR4 material and the thickness is $1 \mathrm{~mm}$, the line width of $50 \mathrm{Ohm}$ is about $3.8 \mathrm{~mm}$, and the area occupied by the $1 / 4$ wavelength line will be $2435 \mathrm{~mm}^{2}$, considering the distance between the lines and the line, the size is will be more than $300 \times 100 \mathrm{~mm}$.

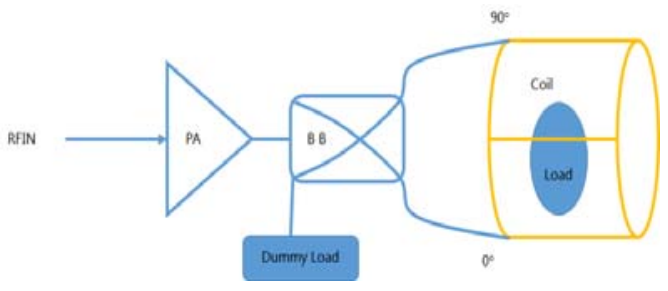

Fig. 1. Power Bridge of MRI.

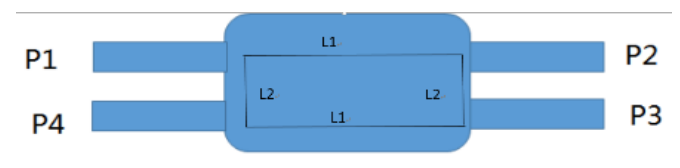

Fig. 2. Typical BB.

Based on the above problems, this paper uses the miniaturized coupling method to realize the bridge, which is realized by microstrip and lumped capacitor, which can reduce the scale of the bridge. this paper use microstrip and capacitor to replace an equivalent $1 / 4$ wavelength line, and can reduce the size of the bridge.

Fig. 3 is a microstrip with capacitor circuit could be realize a $1 / 4$ wavelength line according to the microwave radio frequency circuit theory $[2,3]$, and it could be calculate follow:

$$
\begin{aligned}
& \mathrm{Zp}=\mathrm{Zo} \frac{\mathrm{Zl} 1 \cos \frac{2 \pi \mathrm{l}}{\lambda}+\mathrm{j} Z \operatorname{losin} \frac{2 \pi \mathrm{l}}{\lambda}}{\operatorname{Zoc} \cos \frac{2 \pi \mathrm{l}}{\lambda}+\mathrm{j} \mathrm{Zl} 1 \sin \frac{2 \pi \mathrm{l}}{\lambda}} \\
& Z p=Z s / / C ; Z_{l l}=Z l / / C
\end{aligned}
$$

It's could be difficult to precise calculation the length $\mathrm{L}$ and value of $\mathrm{C}$, and so use the simulation by software and optimization and the process as fig4. This paper design BB for M1.5T MRI system and so the center frequency is $63.87 \mathrm{MHZ}$ [4], and could get the length of microstrip parameters like as $Z_{0}$, length width and thickness and value of capacitor[5,6]. The dielectric material is FR4 and thickness is $1 \mathrm{~mm}$ and permeability is 4.4 .

(1) The microstrip line of $35.26 \mathrm{Ohm}$ width is 3.15 $\mathrm{mm}$ and a length is $130 \mathrm{~mm}$, value of capacitor is $17 \mathrm{pF}$.

(2) The $50 \mathrm{Ohm}$ microstrip width is $1.81 \mathrm{~mm}$, the length is $92 \mathrm{~mm}$, and the capacitance on both sides is 20 $\mathrm{pF}$.
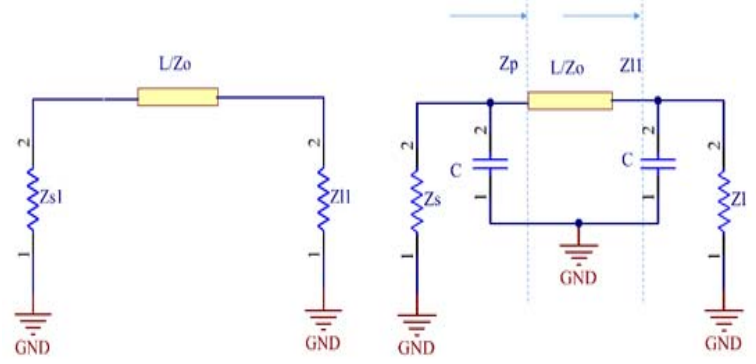

Fig. 3. L of microstrip equivalent circuit.

According to the above calculation, the value of L1 can be obtained as $127 \mathrm{~mm}$.

Set the working frequency to $64 \mathrm{MH}$ and load impedance $\mathrm{Z} 0=50 \mathrm{Ohm}$ observe the $\mathrm{S}$ parameters of the $3 \mathrm{~dB}$ bridge like as fig.4(b), and the simulation model like as fig.4(c). This bridge include a PCB board with 8 capacitors, total has four runners of microstip with some $\mathrm{Z}_{0}$ by Fig4.(c),(b), and the four segment microstrip lines lay up on both sides top and bottom layer.

Like as fig.2, P1 is the input port, $\mathrm{P} 4$ is the isolated terminal, P2 and P3 are the output terminals, and P3 and $\mathrm{P} 2$ are 90 degrees difference phase. The simulation result is shown in Figure 4(d). It can be seen that the design has better performance, the $\mathrm{S} 11<-35 \mathrm{~dB}$, the $\mathrm{S} 41<-$ $35 \mathrm{~dB}$, and the amplitude and phase of the output are close to the ideal result. The bridge is layout and it is fabricated by FR4 material and with ATC 100C capacitor integrated like as fig.5 (a,b,c,d).

Test above MBB with E5071C and the result is following fig.6 $(\mathrm{a}, \mathrm{b})$.

When the operating frequency is $64 \mathrm{MHz}$, the simulation and measured data are compared as shown in Table 1.

Since the micro-strip width of the bridge is greater than $3 \mathrm{~mm}$, the thickness of the copper skin is 1 ounce, and the current that can be passed is greater than $3.5 \mathrm{~A}$ [4]. When the power is $500 \mathrm{~W}$, the voltage of the microstrip line is $158 \mathrm{~V}$, considering the load is $50 \mathrm{Ohm}$, The current is calculated to be $500 / 158=3.16 \mathrm{~A}$ [4], so the continuous wave withstand power can be greater than $500 \mathrm{~W}$. The peak power can withstand a power of more than $5000 \mathrm{~W}$ with a $10 \%$ duty cycle. 


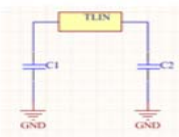

(a)

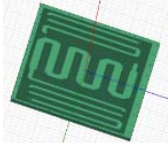

(c)

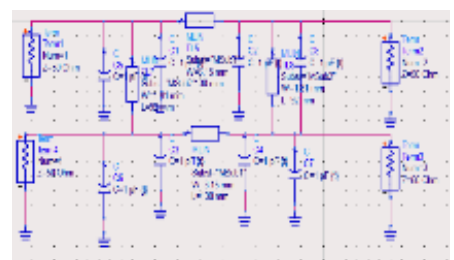

(b)
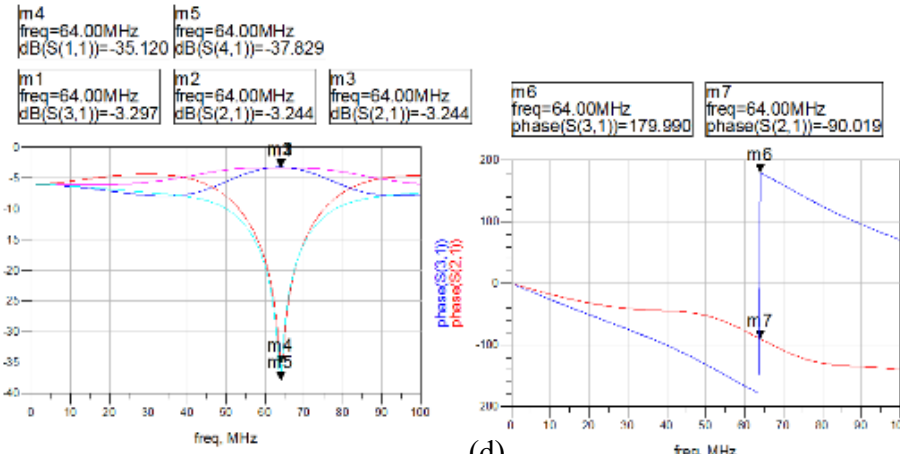

(d)

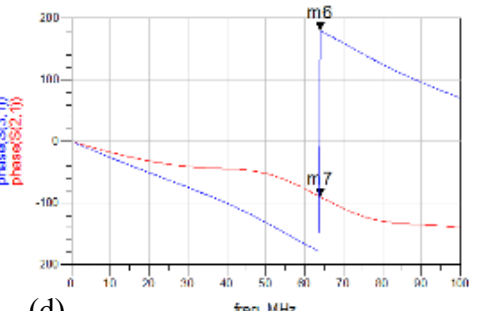

tos, $\mathrm{MHz}$

Fig. 4. (a) Equivalent circuit; (b) Simulation model; (c) Schematic circuit; (d) Simulation data.

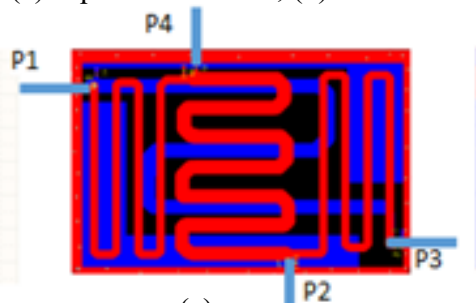

(a)

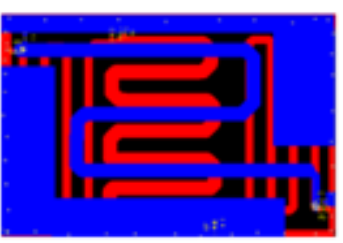

(b)

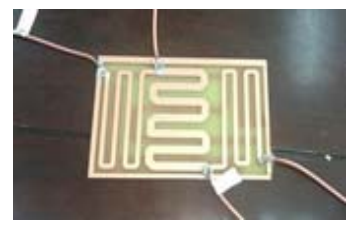

(c)

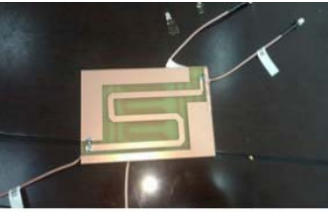

(d)

Fig. 5. (a) PCB layout Top layer; (b) PCB layout bottom layer; (c) PCB top layer; (d) PCB bottom layer.
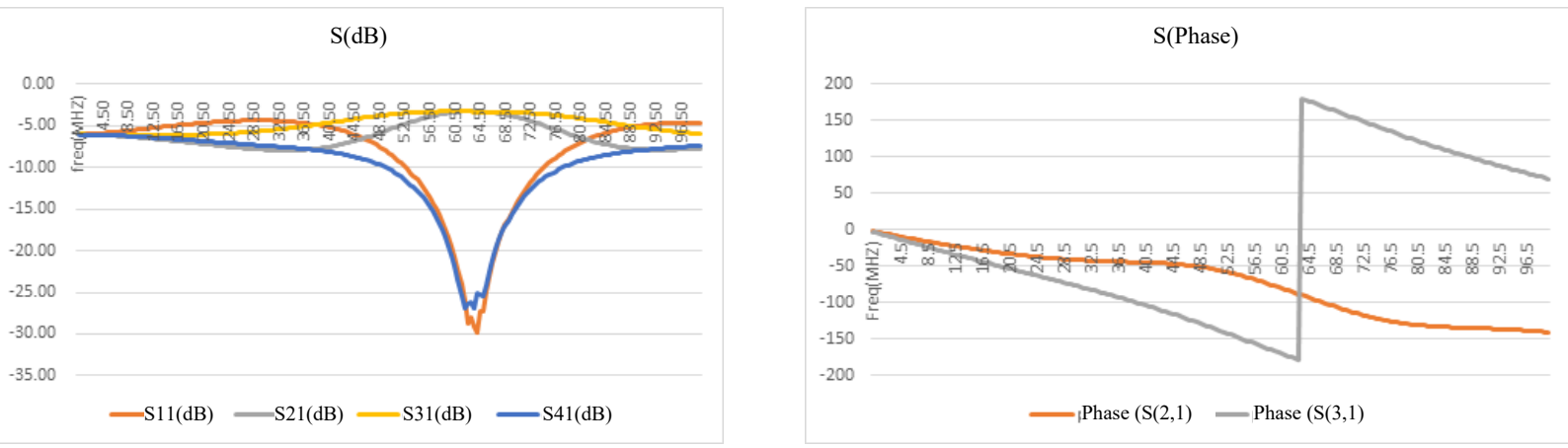

Fig. 6. (a) VSWR anđsi1(dB) (b) Phase gap.

Table 1. Comparison of S-parameter simulation and actual test.

\begin{tabular}{|l|l|l|l|l|l|l|l|l|}
\hline \multirow{2}{*}{ Unit } & \multicolumn{2}{|l|}{ S11 } & \multicolumn{2}{l|}{ S21 } & \multicolumn{2}{l|}{ S31 } & \multicolumn{2}{l|}{ S41 } \\
\cline { 2 - 9 } & Simula-tion & test & Simula-tion & test & Simula-tion & test & Simulation & test \\
\hline $\mathrm{dB}$ & -35 & -29 & 3.3 & 3.33 & 3.29 & 3.2 & -35 & -29 \\
\hline $\operatorname{Phase}\left({ }^{\circ}\right)$ & & & -91 & -89 & 179 & 180 & & \\
\hline
\end{tabular}

\section{Balance bridge experimental magnetic resonance system study}

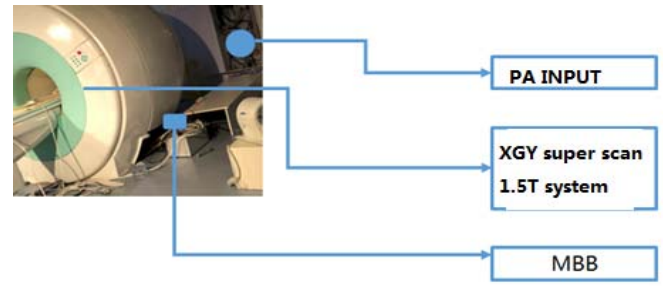

Fig. 7. MBB image test bench of XGY system.
According to the above analysis, in order to verify the imaging characteristics and efficiency characteristics of the bridge, the bridge was placed in a $1.5 \mathrm{~T}$ superconducting magnetic resonance system, and the magnetic resonance system was used by Xingaoyi Medical Equipment Co., Ltd. Super SCAN 1.5T device. Figure 7 shows the test environment. According to the requirements of Xingaoyi equipment system, first, the spectrometer system generates $-12 \mathrm{dBm}$ signal to the RF power amplifier. The output signal of the RF power amplifier can be obtained as $60 \mathrm{dBm}(\approx 1 \mathrm{~kW})$. The signal enters the MBB through the cable, and the MBB divides the 2 signals into the RF coil polarization. The specific experimental data is as shown in table 2 . 
Table 2. Image test result.

\begin{tabular}{|c|c|c|c|c|}
\hline Electric Bridge & $90^{\circ}$ flip angle time & Polarization efficiency & Isolation (S14) & SNR \\
\hline MBB & $1.2 \mathrm{~ms}$ & $99.00 \%$ & $-25 \mathrm{~dB}$ & 100081 \\
\hline XGYBB & $0.9 \mathrm{~ms}$ & $95 \%$ & $-20 \mathrm{~dB}$ & 90065 \\
\hline
\end{tabular}

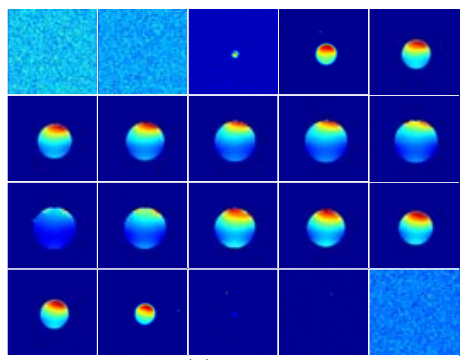

(a)

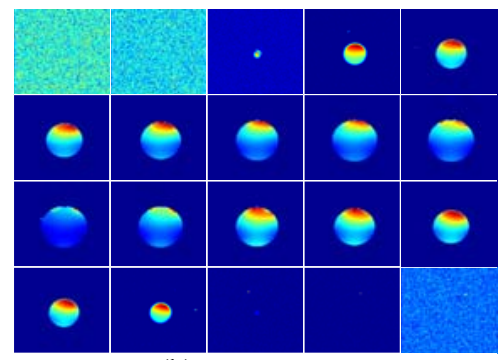

(b)

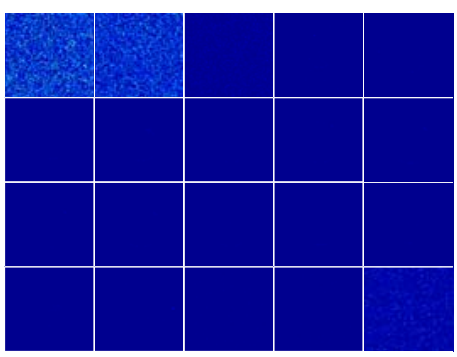

(c)

Fig. 8. (a) MBB SE Imaging; (b) XGYBB SE Imaging; (c) MBB Pixel-XGYBB Pixel.

Fig. 8 is the MRI system test(XGY superscan system) result, (a) is MBB, (b) is the $\mathrm{XGYBB}$ (system orignal device), and (c) is the calculate restult (pixel substration). The MBB's pixel is large than XGYMBB by Fig.7(c). Data as table 3.

Table 3. Imaging quality comparison.

\begin{tabular}{|l|l|l|l|}
\hline Option & $\begin{array}{l}\text { MBB } \\
(1 \mathrm{~kW} \text { input })\end{array}$ & $\begin{array}{l}\text { MBB } \\
(1 \mathrm{~kW} \text { input })\end{array}$ & $\begin{array}{l}\text { Pixel } \\
\text { substration }\end{array}$ \\
\hline $\begin{array}{l}\text { MBB } \\
(1 \mathrm{~kW} \text { input })\end{array}$ & 1896 & 2396 & 500 \\
\hline
\end{tabular}

Since the input signal has reached the peak power of $3 \mathrm{KW}$, in the case of an air conditioning environment temperature of 25 degrees, the power is continuously scanned for 1 hour, and the surface temperature of the MBB is detected by a multi-meter and test result as following table4:

Table 4. Temperature of surface comparison.

\begin{tabular}{|l|c|c|c|c|}
\hline Option & $\begin{array}{l}\text { Surface }{ }^{\circ} \mathrm{C} \\
(10 \text { minutes })\end{array}$ & $\begin{array}{l}\text { Surface }{ }^{\circ} \mathrm{C} \\
(30 \text { minutes })\end{array}$ & $\begin{array}{c}\text { Surface }{ }^{\circ} \mathrm{C} \\
(40 \text { minutes })\end{array}$ & $\begin{array}{l}\text { Surface }^{\circ} \mathrm{C} \\
(60 \text { minutes })\end{array}$ \\
\hline $\begin{array}{l}\text { MBB } \\
(1 \mathrm{~kW} \\
\text { input })\end{array}$ & 24.3 & 25.4 & 25.5 & 23.2 \\
\hline $\begin{array}{l}\text { XGYBB } \\
(1 \mathrm{~kW} \\
\text { input })\end{array}$ & 25.6 & 26.4 & 24.5 & 24.1 \\
\hline
\end{tabular}

It can be seen that MBB has almost no heat temperature rise when it is working continuously 1 hours, and it can completely dissipate heat through natural cooling, which is enough to prove that its power capability can reach 1000 watts of peak power, which fully meets the design requirements.

Above two table results could find, for these polarization effective, isolation, SNR hot effective of key performance, MBB is better than XGYBB.

\section{Conclusion}

This paper proposes a microwave hybrid bridge, which firstly optimizes the design from theoretical analysis and simulation. It is processed into a Micro balanced bridge with FR4 material and some of lumped-parameter capacitive elements. It size only is $100 \mathrm{~mm} \times 60 \mathrm{~mm} \times 1 \mathrm{~mm}$. It is test by E5071C and get the balance of amplitude $\pm 0.02 \mathrm{~dB}$, the phase of diffidence $\pm 1^{\circ}$, insert loss of $0.1 \mathrm{~dB}$ and in-band flatness of $0.01 \mathrm{~dB}$ when operational frequency is $64 \mathrm{MHz} \pm 1 \mathrm{MHz}$. Finally, the Micro-bridge $\mathrm{w}$ be integrated into the Xingaoyi 1.5T MRI system and scan with SE(SPIN Echo) pulse sequence, and get the high performance like as SNR, polarization effective , isolation and hot effective compared with XGYBB product, and also has very small size. This device could be applied the high end 1.5T MRI system. Because the MBB is applied in MRI system and it should be withstand more than $1000 \mathrm{~W}$ RF power, the $1000 \mathrm{~W}$ average power is inputted to port1 and detect the power of port 2, port 3 and port 4 , the power of port 4 is about $30 \mathrm{dBm}$, the port 2 and ports 3 are almost $56.7 \mathrm{dBm}$, and the MBB is installed on the surface of a heat sink. After 1 hours, check the temperature and it's about 2 degree centigrade changed and this is thermal equilibrium, so can be inferred this device could withstand about $1 \mathrm{KW}$ average power. This device is taken to integrate imaging experiment, and system configuration is XGY MRI(Xingaoyi Magnet Resonator Imaging) system of Superscan 1.5T system, this system include with the control system, RF(Radio Frequency) amplifier, gradient amplifier, XGY BB(Balance Bridge of system configuration), body coil, etc. The first use the XGY BB take imaging test and get a group data; then replacement the XGY BB by MBB and take imaging test get another group data; the last analyzed the two groups data, find the MBB integrate into the MRI systems and make imaging experiment and could reduce $20 \%$ imaging time from $1.2 \mathrm{~ms}$ to $900 \mathrm{us}$, and it has good efficiency and homogeneity. So, this device has good performance, small size, and also improve the imaging polarization efficiency. 
This paper gets following fundings supports:

(1) XGY medical equipment co. ltd, project: number: NRFPA001

(2) Ningbo Science and Technology Association Enterprise Fund Project: NB13060020.

(3) Xingyi Normal University for Nationalities professor funding project: Research on chassis design of small tobacco field management machine, funding number: 17XYJS09.

\section{References}

1. D. Jaisson, Planar Impedance Transformer, "Transactions on Microwave Theory and Techniques, vol. 47, no. 5, pp. 592-595, May 1999.

2. Richard Li, RF circuit engineering design Electronic Industry Press, 2011.
3. A. Sutono, D. Heo, Yi. Jan, A. Emery Chen, J. Laskar, High-Q LTCC-based passive library for wireless system-on-package (SOP) module development, IEEE Transactions on Microwave Theory and Techniques 49 (2001) 1715-1724.

4. Canoga Ave., Chatsworth, PC Boards, Casco Circuits, Inc., 10039 D, CA 91311. Tel. (818) 8820972.

5. E.Mark Haacke, Magnetic resonance imaging physics and pulse sequence design, Translated by Zeng Xiaozhuang, China Medical Science and Technology Press, p548 562.2007,06

6. Tom Johansen and Viktor Krozer, Analysis and Design of Lumped Element Marchand Baluns, Danmarks Tekniske Informationscenter. Downloaded on November 6, 2009 at 08:41 from IEEE Xplore. Restrictions apply. 